A. K. McConnell ${ }^{1}$

L. M. Romer ${ }^{2}$

\section{Respiratory Muscle Training in Healthy Humans: Resolving the Controversy}

tory findings. Retrospective analysis of the literature suggests that methodological factors have played a crucial role in the outcome of respiratory muscle training studies. We conclude that in most well controlled and rigorously designed studies, utilizing appropriate outcome measures, respiratory muscle training has a positive influence upon exercise performance. The mechanisms by which respiratory muscle training improves exercise performance are unclear. Putative mechanisms include a delay of respiratory muscle fatigue, a redistribution of blood flow from respiratory to locomotor muscles, and a decrease in the perceptions of respiratory and limb discomfort.

\section{Key words}

Review $\cdot$ exercise performance $\cdot$ inspiratory $\cdot$ fatigue $\cdot$ dyspnea

\section{Introduction}

Early studies into the influence of specific respiratory muscle training (RMT) upon exercise performance in healthy young adults suggested that RMT did not have an ergogenic effect $[16,18,33]$. The results of these studies confirmed the hitherto unchallenged assumption that the respiratory system is overbuilt relative to the rest of the oxygen transport system [14]. However, upon closer inspection of these studies methodological inadequacies are revealed that render the conclusions questionable. More recent studies, incorporating rigorous experimental designs, provide convincing evidence that supports the ergogenic effect of RMT $[29,32,39,40,45,48]$. When viewed in its entirety, the literature is beginning to reveal the specific circum- stances under which RMT improves human performance, as well as providing clues regarding the physiological mechanisms that underlie this ergogenic effect. The purpose of this review is to provide: 1) a brief description of the techniques of RMT, 2) an overview of the published literature pertaining to the effect of RMT upon exercise performance and a resolution to the seemingly contradictory nature of this literature, 3 ) an insight into the putative mechanisms underling the ergogenic effect of RMT, and 4) suggestions for future research.

${ }^{1}$ Department of Sport Sciences, Brunel University, Uxbridge, Middlesex, UK ${ }^{2}$ John Rankin Laboratory of Pulmonary Medicine, Department of Population Health Sciences, University of Wisconsin, Madison, USA

Correspondence

Alison K. McConnell · Department of Sport Sciences - Brunel University · Uxbridge, Middlesex, UB8 3PH · UK·Phone: +01895274000 · Fax: + $01895816341 \cdot$ E-mail: alison.mcconnell@brunel.ac.uk 


\section{Techniques of Respiratory Muscle Training}

Studies into the effect of RMT upon exercise performance have used at least one of the following modes of training: 1) voluntary isocapnic hyperpnea, 2) flow resistive loading, and 3) pressure threshold loading. These training modes, their relative merits, and respective influence upon respiratory muscle function are described below.

\section{Voluntary isocapnic hyperpnea}

Voluntary isocapnic hyperpnea (VIH) training requires individuals to maintain high target levels of ventilation for up to $30 \mathrm{~min}$. To prevent hypocapnia subjects may simply re-breathe through a dead space. However, most studies have used more elaborate apparatus that supply supplemental $\mathrm{O}_{2}$ to avoid hypoxemia, while maintaining isocapnia. Training sessions are typically conducted 3 to 5 times per week at $\sim 60-90 \%$ of maximum voluntary ventilation (MVV). Using VIH, several investigators have shown increases in the time to exhaustion during sustained isocapnic ventilation $[6,7,29,44,45]$, maximum sustainable ventilatory capacity (MSVC) [3,24,26], vital capacity (VC) [3], and MVV $[3,26]$.

Voluntary isocapnic hyperpnea is a relatively time consuming (typically 30 min per session) and physically demanding mode of RMT requiring a high degree of subject motivation. It usually requires regulation of gas exchange, which until recently made it difficult to implement outside of the laboratory. However, a proprietary device is now available (Spirotiger ${ }^{\circledR}$, Idiag AG, Volketswil, Switzerland), making the technique more accessible. Although VIH improves indices of respiratory muscle endurance, it does not improve the maximal pressure generating capacity of the respiratory muscles [26]. The influence of VIH is thus confined to the velocity (flow) axis of the force-velocity relationship of the inspiratory muscles [38]. Finally, it is possible that the high ventilatory flows associated with VIH may induce airway drying and trigger bronchoconstriction in those individuals susceptible to exercise-induced bronchoconstriction.

\section{Flow resistive loading}

Inspiratory flow resistive loading (IFRL) requires individuals to inspire via a variable diameter orifice whereby, for a given flow, the smaller the orifice the greater the resistive load. A device incorporating six resistive orifices and a one way expiratory valve is available (Pflex, Respironics Ltd., NJ, USA). Studies utilizing IFRL have reported increases in inspiratory muscle strength in the range of 18 to $54 \%$ [18,26]. A small ( $5 \%)$ but statistically significant increase in total lung capacity has also been observed after IFRL [26]. However, an inherent limitation of IFRL is that inspiratory pressure, and thus training load, varies with flow (according to a power function) and not just to orifice size. Therefore, it is vitally important that breathing pattern is monitored during IFRL if a quantifiable training stimulus is to be provided. In their 1992 meta-analysis of RMT in patients with chronic obstructive pulmonary disease (COPD), Smith et al. [42] concluded that studies employing IFRL in which inspiratory flow was not controlled failed to elicit improvements in inspiratory muscle function. Although a modified flow resistive loading device can be used to control flow [4], such modifications require complex and expensive hardware, making IFRL impractical for routine use.

A novel approach to IFRL, based on the Test of Incremental Respiratory Endurance (TIRE) technique, has been used by some investigators to train the inspiratory muscles [10] and to test the effects of this training upon aerobic power [9]. The TIRE system uses a flow resistive load ( $2 \mathrm{~mm}$ diameter orifice), an electronic manometer attached via a serial interface to a computer, and dedicated software. Initially, the subject performs several sustained maximal inspiratory efforts through the orifice to provide a baseline pressure-time profile. The subject is then presented with a pressure-time profile typically set at $80 \%$ of the maximal effort. The maximum maneuver is then repeated six times with $60 \mathrm{~s}$ recovery between efforts before the resting time is reduced to $45 \mathrm{~s}$. The subject is required to complete another six efforts, whereby the recovery time is reduced to $30 \mathrm{~s}$ and the user repeats the exercise. There are six different levels in all with diminishing recovery times down to $5 \mathrm{~s}$ between breaths. The exercise is terminated when the subject either completes the full range of breathing exercises or falls beneath the reference pressure-time profile.

Incremental IFRL overcomes the primary limitation of resistive loading by setting inspiratory pressure relative to a subject's maximal capacity. However, the functional relevance of incremental IFRL is questionable since sustained maximal inspiratory efforts bear no relation to the dynamic function of inspiratory muscles during whole body endurance exercise. The influence of incremental IFRL is therefore likely to be confined to the force (pressure) axis of the force-velocity relationship of the inspiratory muscles [38]. Furthermore, training sessions are physically demanding and time consuming (a complete training session takes $\sim 30 \mathrm{~min}$ ). A proprietary version of the device is available (RT Trainer ${ }^{\circledR}$, Sunrise Medical, Carlsbad, CS, USA), but its high cost makes it inaccessible to most individual users.

\section{Pressure threshold loading}

Inspiratory pressure threshold loading (IPTL) requires individuals to produce a negative pressure sufficient to overcome a threshold load and thereby initiate inspiration. Threshold loading permits variable loading at a quantifiable intensity by providing near flow independent resistance to inspiration. This type of loading has been achieved with a weighted plunger [13], a spring-loaded poppet valve [8], a solenoid valve [2], and a constant negative pressure system [11]. Training with IPTL increases the maximal force production $[13,20,21,38-41,47-49]$, the maximal velocity [38-41], the maximal rate of shortening $[38,40,41]$, the maximal power output $[38,40]$, and the endurance $[13,21,49]$ of the inspiratory muscles. When high inspiratory flow rates are used, IPTL has been shown to improve the functional characteristics of the inspiratory muscles at both extremes of their force-velocity relationships [38]. In addition, IPTL is both portable and easy to use, with proprietary devices now available (POWERbreathe ${ }^{\circledR}$; Leisure Systems International Ltd., Southam, UK; Threshold ${ }^{\circledR}$, Respironics, Pittsburgh, PA, USA). The most recent entrant onto the market (Powerlung ${ }^{\circledR}$, Powerlung Inc., Houston, TX, USA) has both an inspiratory and expiratory threshold load. To date, there have been no published studies of the ergogenic effects of expiratory pressure threshold training. 
Since the addition of an expiratory load raises intra-thoracic pressure (partial Valsalva) the potential risks to some groups may outweigh the yet unproven benefits.

The availability of a variety of commercial respiratory training devices makes this form of training more accessible than it has been previously. On the face of it, VIH training offers the highest degree of specificity (by mimicking exercise hyperpnea), but it is strenuous and time consuming, and the literature does not suggest that its high specificity makes VIH any more effective than other modes of RMT (see below). The technique of IFRL is unreliable unless it is used in conjunction with feedback on inspiratory flow rate or pressure, and studies reporting improved inspiratory muscle function have implemented strenuous and time consuming training regimens. The reliability and user-friendliness of IPTL is attractive and it also benefits from being relatively easy to implement as a placebo condition for research purposes [3941]. Thus, until the precise mechanism(s) by which RMT exerts its ergogenic effect are understood fully, the training modality should be selected on the basis of the inspiratory muscle adaptations that are sought and the relative merits of the training technique (i.e., cost, ease of use, time required for training, etc.).

\section{Effect of Respiratory Muscle Training upon Exercise Performance}

This section summarizes the published literature examining the influence of RMT upon whole body exercise performance in healthy humans. The summary excludes abstracts, of which there are many, because these frequently lack the methodological detail required for a critical analysis of their findings. The controversy regarding the ergogenicity of RMT hinges around the results of a relatively small number of predominantly early studies. We explore the possibility that the discrepancies in findings between studies may be because of methodological differences.

The first three studies to examine the influence of RMT upon exercise performance all failed to demonstrate an ergogenic effect $[16,18,33]$ and the authors concluded that RMT did not affect human performance. Each study used a controlled design and endurance trained subjects, with two utilizing cycling $[16,33]$ and one running [18] as the exercise modality. In all studies the performance tests were of high intensity ( $\geq 90 \%$ of maximal oxygen uptake $\left[\dot{\mathrm{VO}_{2 \max }}\right.$ ]) and short duration (5.6 to $12 \mathrm{~min}$ ). These studies employed either VIH $[16,33]$ or IFRL [18] as the training modality. All three studies documented improvements in at least one index of respiratory muscle function, but no statistically significant change in exercise performance. Close scrutiny of the methods and results reveals that in all of these studies, subjects in the RMT groups displayed substantial improvements in exercise performance that were rendered statistically non-significant by insufficient statistical power. For example, in the study by Fairbarn et al. [16] time to exhaustion during a fixed intensity ride at $90 \% \mathrm{VO}_{2 \max }$ increased by $25 \%$ in the training group compared with a non-significant $4 \%$ increase in the control group; there was no significant difference between the groups. Hanel and Secher [18] observed an increase in the distance run in $12 \mathrm{~min}$ in both their RMT (8.5\%) and control group (4.1\%), with no significant difference between groups. Subject numbers were low in all three studies; for example, Morgan et al. [33] tested a total of 9 subjects, 4 of which undertook VIH, while 5 acted as controls. The lack of statistical significance in the group mean data in these studies is suggestive of a type 2 error owing to insufficient statistical power, which is most likely a consequence of the small sample sizes.

Retrospective analysis of the literature as a whole indicates that the type of test used to evaluate exercise performance has played a role in judgements regarding the efficacy of RMT. Fixed intensity tests performed at relatively high percentages of $\mathrm{VO}_{2 \max }$ (>85\%), or incremental tests performed to the limit of tolerance (such as those discussed above), have endpoints that are associated with metabolically unsustainable exercise. These tests are normally short in duration and are terminated abruptly by intolerable sensations associated with failure of both oxygen delivery and anaerobic compensatory mechanisms to maintain a metabolic milieu that can sustain muscle contraction. In these tests, there is a relentless requirement to maintain an externally imposed work rate, and subjects have no opportunity to adjust their output in order to maintain a milieu that is sustainable and tolerable (as, for example, in a time trial). Thus, in the absence of an influence of RMT upon $\mathrm{VO}_{2 \max }$, which most studies have shown to be unaffected by RMT $[6,16,18,20,21,25,29,33$, $39,40,44,49$ ], improvements in high intensity and incremental exercise tests are likely to be small, if present at all. Thus, it is vital that studies utilizing performance tests of high intensity possess adequate statistical power to avoid a type 2 error.

In contrast to the negative outcomes of RMT studies utilizing high intensity exercise tests, moderate intensity tests performed to the limit of tolerance appear to be influenced positively by RMT. The first studies to examine the influence of RMT upon performance in such tests documented impressive improvements. For example, $4 \mathrm{wk}$ of VIH prolonged constant-load exercise $\left(64-77 \% \dot{\mathrm{VO}}_{2 \max }\right)$ by $50 \%$ in sedentary [7] and $38 \%$ in physically active [6] individuals. Minute ventilation and blood lactate concentration were reduced in both groups. However, because the endpoints of such tests are determined primarily by lower intensity, unpleasant sensations that accumulate gradually, their reliability is questionable. Furthermore, the lack of control groups in both studies [6,7] undermines the validity of their findings since improvements could be owing to a learning effect, motivation differences, intra-individual variation, or a combination of these factors. Thus, these positive outcomes were insufficiently convincing and the efficacy of RMT remained equivocal; it was not until the latter part of the 1990s that further studies emerged and the debate about RMT was reopened.

Based on their previous findings [6,7], the group working at the University of Zurich attempted to exclude a number of putative mediators of the ergogenic effect of VIH. First, they hypothesized that a reduction in airway resistance may be a mechanism contributing to the increase in endurance time associated with RMT [25]. The mechanism linking RMT to a lowered airway resistance was based upon their previous finding that minute ventilation was lowered following RMT [6,7]. They suggested that this hypoventilation may have resulted in an attenuation of hypocapnia (a bronchoconstrictor stimulus) and hence to a lower airway resist- 


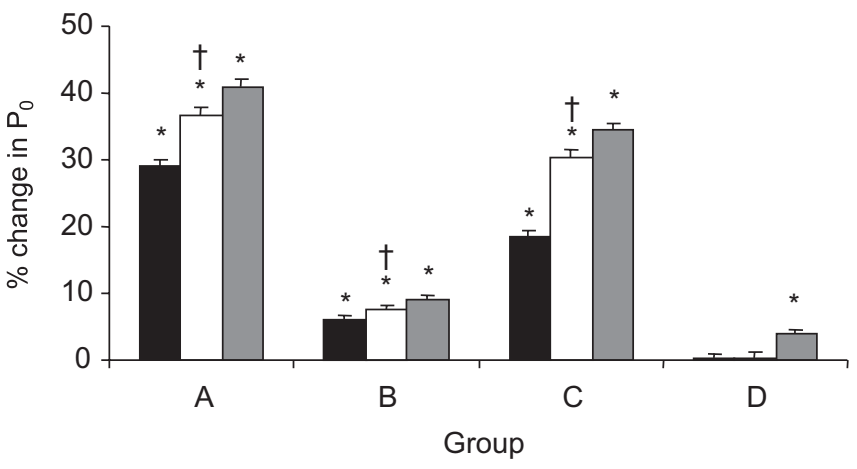

Week $3 \square$ Week $6 \square$ Week 9

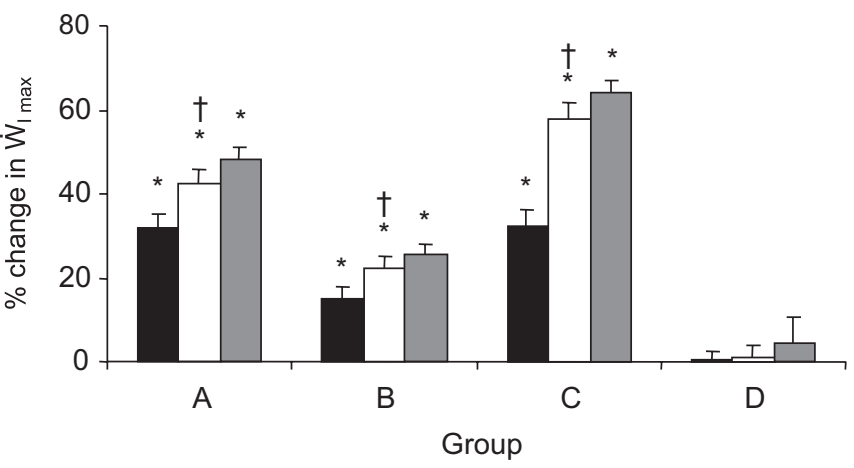

Week $3 \square$ Week $6 \square$ Week 9

Fig.1 Relative changes in maximum dynamic inspiratory muscle function from baseline values for high-pressure RMT (A), high-flow RMT (B), intermediate pressure and flow RMT (C), and control (D) (Mean $\pm \mathrm{SEM}) . \mathrm{P}_{0}$, inspiratory pressure at zero flow; $\dot{\mathrm{V}}_{\max }$, maximum in-

ance, which, they argued, would delay the onset of respiratory muscle fatigue and therefore increase the exercise endurance time. However, the hypothesis was rejected since airway resistance measured immediately after exercise was independent of exercise-induced hyperventilation and hypocapnia. The majority of subsequent studies have found no change in minute ventilation during constant load exercise at identical intensity pre- and post-RMT $[44,45,49]$.

More recently, the Zurich group examined the influence of VIH upon time to the limit of tolerance and blood lactate concentration during a constant load cycling test [44]. Four weeks of VIH increased cycling time by $27 \%$ in a group of 20 healthy subjects. Blood lactate concentrations were reduced by $15 \%$ during maximal incremental exercise and by $8 \%$ during constant load exercise. The authors speculated that the reduction in blood lactate concentration was most likely caused by an improved lactate uptake by the trained respiratory muscles, although reduced exercise blood lactate concentrations per se were unlikely to explain the improved cycling performance after RMT. The authors favored a causal mechanism linked to respiratory muscle fatigue. Unfortunately, like their previous work, this study is flawed by the failure to employ a control group (see below).

Changes in endurance capacity and blood lactate concentrations after RMT are consistent with responses seen after a period of

$\begin{array}{llll}\text { Zeitschrift } & \text { IJSM sm109 } & \text { Satzbetrieb } & \text { Ziegler + Müller } \\ \text { Verlag } & \text { Thieme/Hentze } & \text { Datum } & 24.11 .2003\end{array}$

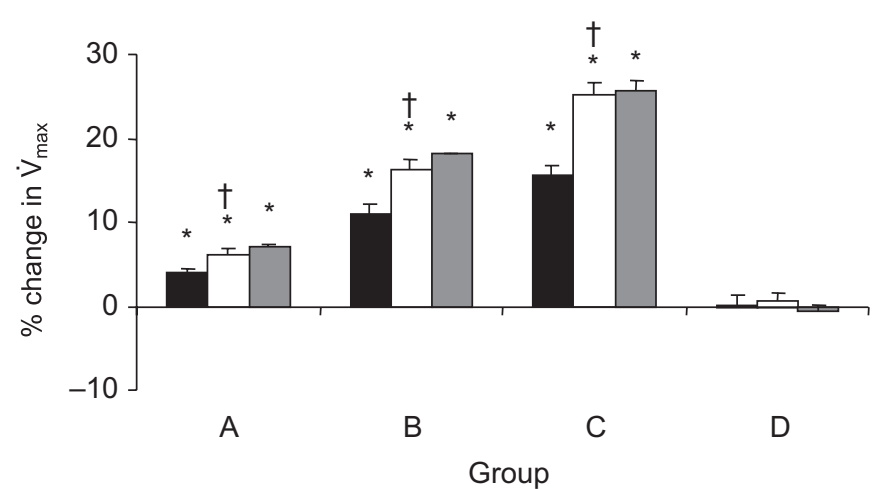

Week $3 \square$ Week $6 \square$ Week 9

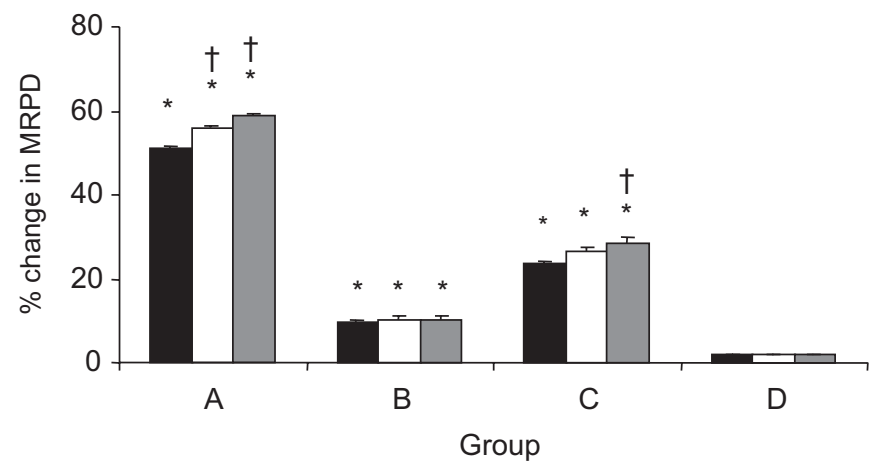

Week $3 \square$ Week 6 -Week 9

spiratory flow; $\dot{W}_{\text {Imax }}$, maximum inspiratory muscle work rate; MRPD, maximum rate of pressure development. Note: * significantly different from baseline $(p \leq 0.05)$; $\dagger$ significantly different from preceding time point $(p \leq 0.05)$. From Romer and McConnell [38].

whole body aerobic training. Markov et al. [29] examined whether the increase in stroke volume and heart rate that accompanies execution of VIH induces a cardiac training effect that improves myocardial performance, and thus whole body exercise performance. Three groups of subjects performed $15 \mathrm{wk}$ of either VIH $(n=13)$, whole body endurance training $(n=9)$, or no training $(\mathrm{n}=15)$. Performance during a breathing endurance test improved significantly in the VIH group, but not the other groups. During an incremental cycling test, peak $\mathrm{VO}_{2}$ increased significantly by $19 \%$ and peak work rate by $22 \%$ only in the endurance training group. Time to exhaustion at $70 \%$ of peak work rate increased significantly by $41 \%$ in the endurance group and by $24 \%$ in the VIH group, but not in the control group. The only statistically significant cardiovascular changes observed during a constant load exercise test at $60 \%$ peak work rate were observed in the endurance-trained group post-training (12\% decrease in heart rate; $17 \%$ increase in stroke volume). The authors concluded that the improved cycling endurance time following $15 \mathrm{wk}$ VIH was not because of a cardiovascular training effect induced by VIH.

The Zurich group have also examined the influence of VIH upon blood gas concentrations [45]. Their rationale was that VIH might enhance the arterial partial pressure of $\mathrm{O}_{2}$ during exercise, thus increasing oxygen supply to the working muscles, evoking an ergogenic response similar to that observed during hyperoxic 
Zeitschrift IJSM sm109

Verlag Thieme/Hentze
Satzbetrieb Ziegler + Müller Datum 24.11.2003 exercise. Two groups of subjects ( $\mathrm{VIH}, \mathrm{n}=13$; non-training control, $\mathrm{n}=15$ ) were assessed before and after a $15 \mathrm{wk}$ period of VIH. However, while there were significant increases in breathing $(633 \%)$ and cycling endurance $(24 \%)$ in the VIH group, there were no changes in the arterial partial pressure or saturation of $\mathrm{O}_{2}$ during exercise. The authors concluded that the improved cycling endurance time could not be attributed to an increased $\mathrm{O}_{2}$ supply to the working muscles. This study addressed a similar hypothesis to that of Inbar et al. [21], who reasoned that some well-trained endurance athletes experience exercise-induced arterial hypoxemia and that respiratory muscle fatigue and relative hypoventilation may be implicated in this process. Using IPTL, they sought to improve respiratory muscle function and therefore aerobic power in these individuals. Although measures of inspiratory muscle strength and endurance increased significantly in the RMT group compared to the control group, there was no change in maximum minute ventilation $\left(\dot{\mathrm{V}}_{\mathrm{Emax}}\right), \dot{\mathrm{V}}_{2 \max }$, breathing reserve (MVV $\left.-\dot{\mathrm{V}}_{\mathrm{Emax}}\right)$, or arterial $\mathrm{O}_{2}$ saturation. Taken together, the findings of Inbar et al. [21] and Stuessi et al. [45] confirm that improved cycling endurance time after RMT cannot be explained by an improvement in the oxygen transport system.

Most recently, the Zurich group found that increased cycling endurance time after RMT ( VIH) was accompanied by a reduction in peripheral chemoreceptor sensitivity [32]. However, there were no accompanying alterations in breathing pattern or minute ventilation during constant intensity exercise after RMT. The authors did not offer an explanation for the role of peripheral chemoreceptor sensitivity in the ergogenic effect of RMT.

Williams et al. [49] used an alternative approach to RMT of inspiratory pressure threshold loading (IPTL). Their uncontrolled trial of RMT in 7 collegiate distance runners found statistically significant increases in inspiratory muscle strength (MIP) and endurance (time to exhaustion at 60\% MIP) after $4 \mathrm{wk}$ of RMT. They observed no change in performance during an incremental treadmill test to exhaustion, a finding that is consistent with previous studies $[6,16,18,20,21,25,29,33,39,40,44]$. However, they also noted no change in treadmill running time to exhaustion at a fixed intensity of $85 \% \mathrm{VO}_{2 \max }$. Neither did they observe any significant changes in heart rate, oxygen uptake, minute ventilation, or blood lactate concentration during the fixed intensity run. The duration of the fixed intensity test $(\sim 19 \mathrm{~min})$ was similar to that of studies that have shown an ergogenic effect $[6,7,44]$, although these studies employed different exercise modalities (cycling and rowing). Boussana et al. [5] suggested that the crouched posture in cycling (and perhaps also rowing) makes this modality more challenging to the inspiratory muscles than running, which may influence the efficacy of RMT (see Potential Mechanisms). Williams et al. [49] did, however, note a tendency $(\mathrm{r}=-0.650, \mathrm{p}=0.057)$ towards an inverse relationship between the reduction in dyspnea during the final minute of the endurance run and the improvement in MIP, which is consistent with the findings of other investigators utilizing IPTL [39,40,48]. Nevertheless, the small number of subjects, and the absence of a control group and reliability data for the running test, all undermine the authors' conclusion that RMT is not an effective adjunct to whole body training in endurance athletes. Clearly, further research is required to determine the influence of exercise modality upon the ergogenicity of RMT.
The studies with positive outcomes described so far have used performance tests that required subjects to cycle to the limit of tolerance at a fixed, supra-lactate threshold intensity. Only one study has documented an improvement in maximal incremental exercise performance following RMT [9]. Chatham and colleagues assigned 21 subjects at random to either a RMT group (TIRE at $80 \%$ of sustained maximal inspiratory pressure, 3 times per week), or a control group who received no training during the intervention period. Following training the RMT group (but not the control group) significantly increased inspiratory muscle strength and endurance, as well as $\dot{\mathrm{V}}_{2 \max }$ estimated from the number of shuttles completed during a progressive shuttle run test. Since no study of RMT has ever documented an increase in directly measured $\mathrm{VO}_{2 \max }$, Chatham et al.'s data suggest that the RMT group were better able to sustain exercise at their $\dot{\mathrm{VO}}_{2 \max }$, rather than exhibiting an increase in maximal aerobic power per se. Regardless, the design of the study can be criticized for not controlling the influence of the subjects' expectations postRMT. Subjects in both groups may have been aware of the stage achieved during the pre-RMT test, but only the RMT group would have had the expectation of improving their performance postRMT; thus their motivation to improve may have been higher. A more recent, double-blind study [39] failed to support Chatham et al.'s finding of improved incremental shuttle running performance.

The use of constant intensity exercise tasks or maximal incremental tests performed to the limit of tolerance allow investigators to examine physiological changes under identical conditions pre- and post-RMT. Although such study designs have high internal validity, they lack the external validity that arises from performance tests that mimic competitive situations. They also appear to have generated conflicting findings with respect to the efficacy of RMT as an ergogenic aid. More recent studies have assessed the efficacy of RMT for competitive athletes using simulated time trial performance as the outcome measure. It might be argued that the high external validity of this approach provides a greater degree of importance to the findings of such studies.

Volianitis et al. [48] were the first to examine the effect of RMT (IPTL) upon simulated time trial performance. Fourteen competitive female rowers were assigned at random to either a RMT group (50\% MIP, 30 breaths twice daily) or a placebo group (15\% MIP, 60 breaths $\left.\cdot \mathrm{d}^{-1}\right)$. Eleven weeks of RMT significantly improved MIP by $45 \%$, the distance covered in $6 \mathrm{~min}$ by $3.5 \%$ $(52 \mathrm{~m})$, and $5000 \mathrm{~m}$ time trial performance by $3.1 \%$ (36 s) in the RMT group; the placebo group experienced much smaller, nonsignificant changes in these variables. The RMT group experienced less inspiratory muscle fatigue following the 6 min maximal effort compared to pre-RMT values (- 3\% MIP versus - 11\% MIP, respectively). Furthermore, there was a reduction in the perception of dyspnea and blood lactate concentration during an incremental exercise test, but only the former achieved statistical significance when comparisons were made with the placebo group. Although the intensity of the time trials was high, performance improved significantly in both tests $(6$ min piece and $5000 \mathrm{~m}$ ), which suggests that it may not be the high intensity of incremental and fixed high intensity exercise per se that renders them insensitive to the effects of RMT (see later). 


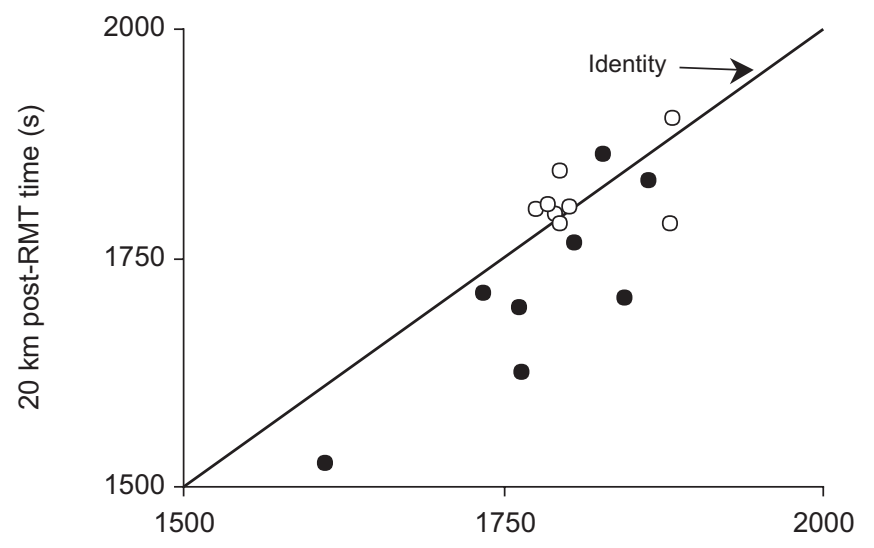

a

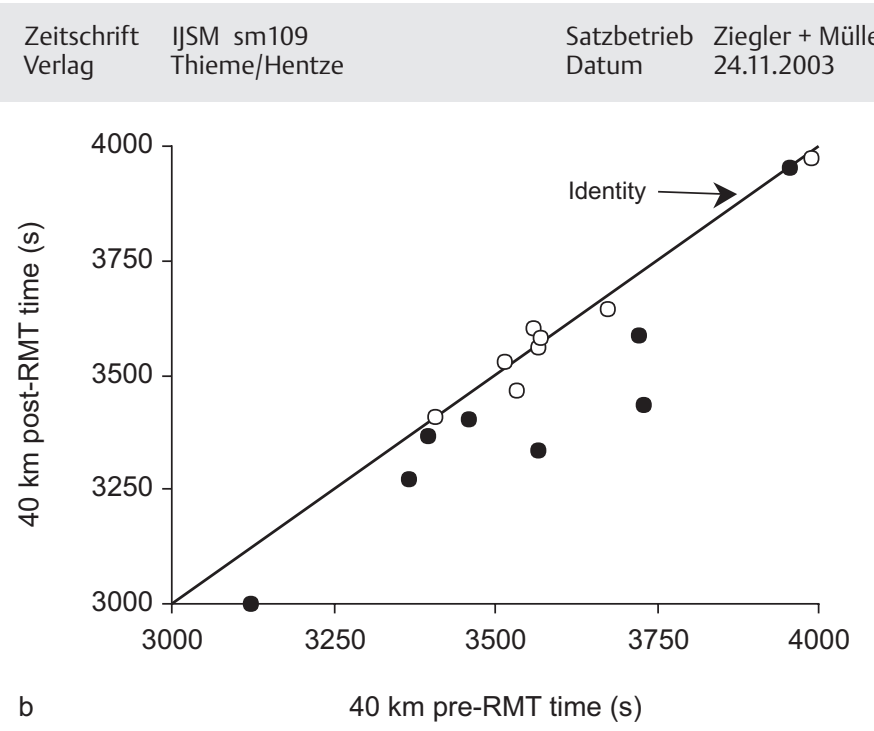

(open symbols) groups. Note that 20 and $40 \mathrm{~km}$ time-trial performances were shorter in 7 of 8 post-RMT trials. From Romer et al. [40].

Fig. $\mathbf{2}$ a and $\mathbf{b}$ Relationship between pre-RMT and post-RMT $20 \mathrm{~km}$ (a) and $40 \mathrm{~km}$ (b) cycling time-trial performance for pressure-threshold inspiratory muscle training (closed symbols) and sham-training control

Most of the studies discussed so far contain methodological inadequacies that render the findings equivocal. In a recent study published in two parts $[40,41]$ our group attempted to account for the major flaws in previous work by designing a study that was rigorously controlled. Most studies have failed to quantify accurately the whole body training and RMT undertaken by the subjects, which is unfortunate considering the strong dose-response relationship of RMT $[39,51]$. Other studies have risked type 2 errors by using inappropriate tests of exercise performance, tests with unquantified reliability, small sample sizes, or a combination of these factors $[6,7,9,16,18,21,25,29,32,33,44$, $45,49]$. Some investigators have risked subject bias by failing to use control groups $[6,7,44,49]$ or placebo groups $[9,16,25,29$, $32,33,45]$. In studies where placebo groups were used, the treatments were not presented to subjects in a double-blind manner $[18,21,43,48]$.

In an attempt to address some of the inadequacies of previous studies, Romer et al. examined the influence of $6 \mathrm{wk}$ RMT (IPTL) upon simulated time trial performance [40] and exercise-induced inspiratory muscle fatigue [41] in 16 competitive male cyclists using a double-blind, randomized, controlled design. Subject numbers were determined by a priori power calculations, whole body exercise training was quantified using a training impulse score (TRIMP), and compliance of RMT was quantified electronically using a logging system incorporated into the training device. Eight subjects undertook RMT, while 8 served as controls undertaking sham RMT. The TRIMP scores showed no change in either group post-RMT and compliance to the prescribed RMT regimens was $>95 \%$ in both groups. Global inspiratory muscle function was quantified by defining the inspiratory pressureflow-power relationships. All parameters of inspiratory muscle function increased significantly post-RMT in the experimental group, but not the placebo group. The RMT group also showed greater improvements in time trial performance compared with the placebo group (3.8\% and $4.6 \%, 20 \mathrm{~km}$ and $40 \mathrm{~km}$, respectively) (see Fig. 2) and an attenuation of the exercise-induced inspiratory muscle fatigue. Perceptions of both limb discomfort and dyspnea were significantly lower in the RMT group posttraining. However, there were no changes in maximal incremen- tal exercise performance. This is the most rigorously designed trial of RMT to date, and it confirms that time trial performance is improved after RMT in the absence of a change in maximal incremental exercise performance.

The difference between time trial exercise and the other forms of intense activity may be the self-imposed nature of the exercise intensity during a time trial. The subjects push themselves to work as hard as they feel able and this may permit them to "express" the benefits of RMT (for example, reduced effort perception) by going faster. In contrast, an incremental or high intensity, fixed workload test cannot be influenced by the subject in any way other than by prolonging the exercise to a point where the energy supply systems have reached the point of failure. It is clear that any ergogenic influence exerted under the latter conditions will be small, and hence difficult to measure. This may help to explain at least some of the apparent contradictions between studies.

It should be clear from the preceding discussion that studies of RMT require rigorous designs and sensitive performance tests in order to detect the ergogenic influence of the intervention. A final factor that is worth mentioning in the context of study design is that of the RMT itself. The results of a recent study by Sonetti et al. [43] highlight several methodological issues that are relevant to the design of future studies. Those authors used the time to exhaustion during a constant load cycling test, the time to complete a simulated $8 \mathrm{~km}$ time trial, and the maximal power achieved during a maximal incremental exercise test as outcome measures. The study is unique in that it exposed the subjects to concurrent IPTL and VIH. After 5 wk of RMT the authors noted a significant improvement in exercise performance in competitive male cyclists, but this was not significantly different from the improvement observed in their placebo group. The placebo control consisted of $30 \mathrm{~min} \cdot \mathrm{d}^{-1}$ breathing through an IPTL device packed with gravel (to convince the subjects that they were undertaking hypoxic training). There was a significant $7.7 \%$ increase in MIP and an "extremely variable", non-significant $74 \%$ improvement in a test of breathing endurance in the RMT group. The placebo group demonstrated a 3.7\% increase in MIP and a 
77\% increase in breathing endurance. Performance in the constant load cycling test increased significantly in the RMT group (26\%) and the placebo group (16\%), as did maximal incremental exercise performance ( $8.6 \%$ and $6.3 \%$, respectively). Time trial performance ( $13 \mathrm{~min}$ ) improved significantly in the RMT group ( $1.8 \%)$, but not the placebo group ( $0.3 \%)$. None of the changes in respiratory muscle function and exercise performance were significantly different between groups. The authors did not observe any changes in heart rate, ventilation, blood lactate concentration, or dyspnea at equivalent time points during the constant load exercise test.

The changes in inspiratory muscle function noted by Sonetti et al. were much smaller than those observed by other investigators $[21,29,39-41,44,45,48,49]$. It is possible that the concurrent training regimen, in which the respiratory muscles were subjected simultaneously to both a strength (IPTL) and an endurance training (VIH) stimulus, resulted in sub-optimal adaptations in response to both. This phenomenon is well established for peripheral skeletal muscles [28]. Furthermore, the improvements in respiratory muscle function and exercise performance observed in the placebo group suggest that they experienced an RMT endurance training effect (owing to the small inherent resistance of the placebo device). It is therefore unsurprising that non-significant differences in exercise performance were found between the groups, since both benefited from a training effect. Although incremental exercise performance and cycling time to exhaustion improved in both groups, time trial performance only improved in the group who received a strength-training stimulus (i.e., the training group). To date, the influence of VIH alone has not been assessed using time trial performance as an outcome measure, so its influence on this type of performance remains unknown.

In the context of future study design, a study by Hart et al. [20] provides some cautionary observations and once again highlights the importance of selecting appropriate outcome measures. Those authors sought to evaluate the influence of $6 \mathrm{wk}$ RMT (IPTL) upon inspiratory muscle function and exercise performance in healthy adult subjects. They observed a small (12\%) increase in MIP post-training in their 6 training subjects, which was statistically different from their sham-training group. However, the change was much smaller than that noted by other investigators employing IPTL $[21,39-41,48,49]$, and is suggestive of poor training compliance. In addition to MIP, the authors used a non-volitional measure of diaphragm function, twitch transdiaphragmatic pressure, in an effort to more rigorously assess the RMT-induced changes in diaphragm function. They noted no statistically significant change in twitch pressure in either group and concluded that the improvement in MIP was a task learning effect and that there had been no improvement in inspiratory muscle function. However, their own power analysis revealed that the reliability of twitch transdiaphragmatic pressure was so poor that it would have required 234 subjects to detect a $10 \%$ effect. Their conclusion that IPTL does not improve inspiratory muscle function was therefore based upon a flawed statistical approach. Unfortunately, the authors also used an inappropriate outcome measure of exercise performance (i.e., time to exhaustion during an incremental exercise test), and it was therefore not surprising that they also observed no change in performance

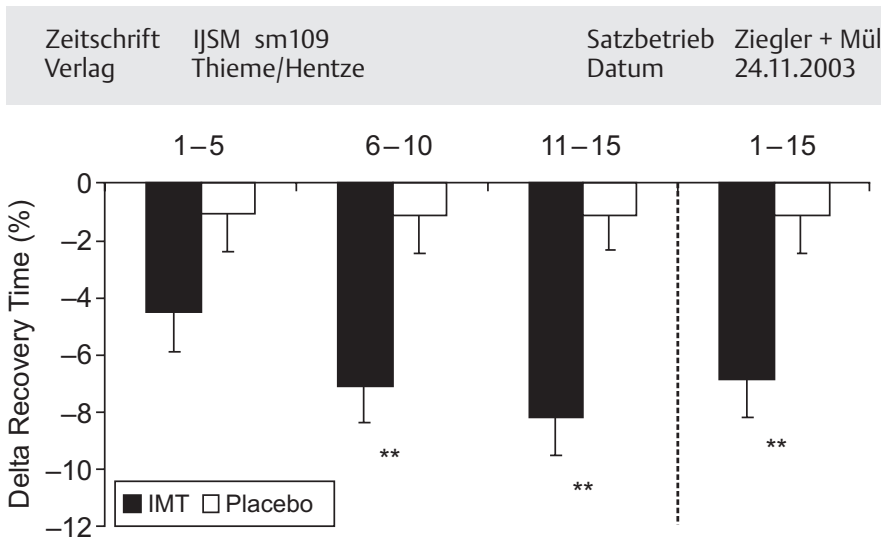

Repetition

Fig. 3 Changes in cumulative recovery time relative to baseline during a repetitive sprint test for pressure-threshold inspiratory muscle training (closed bars) and sham-training control (open bars) groups (Mean \pm SEM). Note: ${ }^{* *}$ significantly different between RMT and control $(p \leq 0.01)$. From Romer et al. [39].

post-RMT. Readers are referred to a detailed critique of Hart et al.'s study that has been published elsewhere [31].

To date, all studies of RMT have assessed its influence upon endurance exercise performance. In a novel experiment, Romer et al. [39] examined the influence of RMT upon repeated sprint performance in a double-blind placebo-controlled study on 24 repeated sprint athletes. Those authors reasoned that the attenuation of dyspnea associated with RMT might enhance the perceived rate of recovery during a repeated sprint test (15 maximal sprints of $20 \mathrm{~m}$ ) that incorporated self-determined recovery periods. Following 6 wk of IPTL, there was a 31\% increase in MIP and a $6.2 \%$ reduction in the total recovery time between sprints (see Fig. 3), with no change in actual sprint performance. The data suggest that the subjects' perception of their recovery was enhanced post-RMT. There was also a significant reduction in blood lactate concentration and perceptual responses to a sub-maximal, fixed intensity running test. There were also significant correlations between the changes in recovery time and the changes in effort perceptions ( $r^{2}=0.57$ for limb discomfort; $r^{2}=0.41$ for dyspnea), and blood lactate concentrations $\left(r^{2}=0.52\right)$. The close relationship between changes in performance and changes in blood lactate concentrations is consistent with previous findings [44]. However, there was no change in progressive shuttle running performance, which is in contrast to the findings of one previous study [9].

In summary, a clearer picture is beginning to emerge from the seemingly conflicting results within the literature. Early studies with negative findings were hampered by weak experimental designs and insufficient statistical power $[16,18,33]$. In addition, some experimenters $[20,21]$ used performance tests that other studies showed to be insensitive to RMT, while some may have failed to observe significant changes in performance because of inadequate training stimuli $[20,43]$, training effects in the placebo group [43], or both. Although the literature supporting the ergogenic effect of RMT is far from perfect, the majority of recent studies have been designed rigorously and the data now provide convincing support for an ergogenic effect of RMT during moderate fixed intensity exercise and time trial performance. The latter has the greatest external validity and relevance for athletic performance. 
The physiological parameters that are most often associated with improved exercise performance following RMT are attenuated blood lactate responses $[6,7,25,39,44]$ and reduced effort sensations [39, 40,46, 48, 49]. Indeed, Spengler et al. [44] and Romer et al. [39] have demonstrated a significant association between post-RMT changes in blood lactate concentration and performance improvement, such that up to $52 \%$ of the variation in performance can be accounted for by the changes in lactate concentration [39]. Although this relationship is not likely to be "cause and effect", it suggests that lactate turnover, or something related to it, is linked strongly to the ergogenic effect of RMT.

If lactate turnover is influenced by RMT, it is reasonable to question why incremental and fixed high intensity tests are insensitive to RMT. Physiologically, high intensity exercise is limited by the inability of the oxygen transport system to maintain ATP resynthesis, and the associated accumulation of anaerobic metabolites that lead to muscle contraction failure and intolerable discomfort. It is reasonable to suppose that even if RMT were to have a positive influence upon lactate turnover that this would be overwhelmed in the face of the rapidly escalating metabolic acidosis that accompanies incremental or fixed high intensity exercise. In contrast to the abrupt catastrophic failure of these tests, fixed intensity tests of moderate intensity (typically, 20 to 30 min duration) are limited by a constellation of inputs including gradual increases in blood lactate accumulation and perceived exertion. The ability to maintain a quasi steady state of these limiting factors for longer, or at a slightly higher intensity of work post-RMT may be what mediates the ergogenicity of RMT in moderate intensity tests. But what physiological changes enable this latter scenario to be realized, and why are attenuated blood lactate concentration and effort sensations associated with improved performance following RMT? The following section attempts to piece together evidence from a number of sources to shed light on these questions.

\section{Potential Mechanisms}

The precise mechanisms by which RMT improves whole body exercise performance are unclear. It appears that central circulatory adaptations, such as increases in maximal stroke volume or cardiac output, do not occur with RMT [29]. Therefore, it is likely that changes within the respiratory muscles underlie the ergogenic effect of RMT, although they may ultimately involve interactions with the brain or working locomotor muscles. Increases in the maximal force production, the maximal velocity of shortening, and the endurance of the respiratory muscles have been observed consistently with RMT (see "Techniques of Respiratory Muscle Training"). The functional significance of an improvement in respiratory muscle function might be to prevent or delay exercise-induced diaphragmatic fatigue, which is known to occur in healthy subjects with a range of fitness levels [22]. Evidence from animal models suggests that whole body training improves inspiratory muscle function [34], which, it might be argued, renders RMT redundant. However, available evidence suggests that highly trained individuals are not resistant to inspiratory muscle fatigue $[1,41,48]$. This most likely arises because the improvements in inspiratory muscle function due to whole body training are offset by the increase in ventilatory demand that ac- companies an improved aerobic capacity, thus negating the potentially protective effect of the training-induced inspiratory muscle adaptations. Recent evidence suggests that RMT attenuates the decrease in global inspiratory muscle function after both short-term (6 min), high-intensity rowing [48] and longer duration ( $\sim 30-60 \mathrm{~min})$, lower intensity $(\sim 75-82 \%$ peak power $)$ cycling exercise [41] in highly trained women and men, respectively.

Evidence is now available in humans that RMT induces structural adaptations within the inspiratory muscles. Specifically, Ramírez-Sarmiento et al. [35] have used open biopsies of the external intercostal muscles of patients with COPD to evaluate RMT-induced adaptations. Fourteen patients were assigned at random to either a RMT group (IPTL, $40-50 \% \mathrm{MIP}, 30 \mathrm{~min} \cdot \mathrm{d}^{-1}, 5 \mathrm{~d} \cdot \mathrm{wk}^{-1}$, $5 \mathrm{wk}$ ) or a sham training control group. Biopsies from external intercostal muscles and vastus lateralis (control muscle) were taken before and after the training period. Statistically significant increases in the proportion of type I fibers (38\%) and the size of type II fibers (21\%) were observed in the RMT group. These morphological changes were associated with increases in both the strength and endurance of the inspiratory muscles. No changes were found in either the sham training control group or the control muscle. These structural changes within the inspiratory muscles are consistent with increased fatigue resistance since they may reduce the force contribution from each active myofiber or the number of myofibers at a given submaximal level of ventilation. In conjunction, a stronger type I fiber may allow individuals to delay recruitment of the less efficient type II fibers. The resultant resistance to fatigue of the respiratory muscles would result in an overall improvement in cellular homeostasis.

The avoidance of inspiratory muscle fatigue may be crucial in mediating the ergogenicity of RMT. Recent evidence suggests that reflex activity from chemo-sensitive type III/IV receptors of fatiguing respiratory muscles may induce sympatheticallymediated reflex vasoconstrictor activity in the limbs (for a review see Romer and Dempsey [37]). Delaying, or diminishing the magnitude of respiratory muscle fatigue may attenuate reflex activity from type III/IV receptors of these muscles and perhaps reduce sympathetic vasoconstrictor activity in the limbs. It is possible that the concomitant increase in limb blood flow would increase $\mathrm{O}_{2}$ delivery to the limbs and decrease the circulating blood lactate concentration. These changes would likely lead to reductions in both limb fatigue and peripheral effort sensations, and perhaps an increase in the ability of the limbs to perform work. Attenuation of sensory input to the central nervous system would be expected to occur through: 1) reduced inhibitory feedback from fatiguing respiratory muscles [17], 2) an alteration in the pattern of tension development [15], 3) a smaller fraction of maximum tension generated with each breath [36], or a combination of these factors. A decrease in the perceptual response to exercise after RMT might also be through the repeated generation of large respiratory pressures during RMT, which may have a desensitizing effect upon the sensory input from the respiratory muscles to the brain [50]. 


\section{Conclusions}

A review of the literature reveals that in well controlled and designed studies, using appropriate performance outcome measures, RMT has a positive influence upon exercise performance during both moderate fixed intensity exercise tasks and simulated time trials ranging from 6 min duration [48] to $1 \mathrm{~h} \mathrm{[40].} \mathrm{The}$ literature does not support an effect of RMT upon maximal incremental exercise. An attenuated blood lactate response to exercise is an occasional finding, and the amelioration of dyspnea and exercise-induced inspiratory muscle fatigue, are commonly observed. One study has also shown a beneficial influence of RMT upon recovery time during a repetitive sprint test. Carefully conducted studies have shown that the ergogenic effect of RMT cannot be ascribed to a central cardiovascular training effect, or to any change in the capacity of the oxygen transport system. The importance of experimental design is illustrated by several studies that have failed to observe statistically significant improvements in exercise performance because of type 2 errors, inadequate RMT protocols, training effects in the placebo group, or a combination of these factors.

Uncertainty still exists concerning the mechanisms governing the ergogenic potential of RMT, but the avoidance of respiratory muscle fatigue and its systemic and perceptual repercussions may play a crucial role. Thus, it is likely that the ergogenic effect of RMT has a multifactorial etiology that may include: 1) the direct effect of RMT upon respiratory muscle fatigue, 2) RMT's indirect effects upon improving blood flow distribution to limb locomotor muscles in heavy exercise, and 3) RMT's direct and indirect effect upon the intensity with which both respiratory and peripheral efforts are perceived.

\section{Future Directions}

The need for some key experiments in several areas covered here may be obvious. Answers to the following questions would help greatly.

1. What are the specific aspects of respiratory muscle function enhancement that are linked to ergogenic effects in athletes?

2. What are the mechanisms by which improvements in respiratory muscle function and fatigue resistance improve exercise performance?

3. Are the mechanisms by which RMT improves exercise performance dependent upon the training modality (for example, IPTL vs. VIH)?

4. What is the role of expiratory muscle fatigue in exercise tolerance and does specific expiratory muscle training enhance exercise performance?

5. Does the dual action of the respiratory muscles in certain sports (for example, rowing, swimming, and kayaking) make participants in these sports more sensitive to the ergogenic effect of RMT?

6. A high work of breathing and substantial metabolic consequences are expected to occur during strenuous exercise in specific populations (for example, healthy fit elderly [23] and fit young female subjects [30]) and environmental conditions (for example, heat [19] and extreme partial pressures of $\mathrm{O}_{2}$
Zeitschrift IJSM sm109

Verlag Thieme/Hentze

Satzbetrieb Ziegler + Müller

Datum 24.11.2003

$[12,27])$. Is RMT particularly beneficial for performance in these populations and environmental conditions?

\section{References}

${ }^{1}$ Babcock MA, Pegelow DF, Johnson BD, Dempsey JA. Aerobic fitness effects on exercise-induced low-frequency diaphragm fatigue. J Appl Physiol 1996; 81: $2156-2164$

2 Bardsley PA, Bentley S, Hall HS, Singh SJ, Evans DH, Morgan MD. Measurement of inspiratory muscle performance with incremental threshold loading: a comparison of two techniques. Thorax 1993; 48: $354-359$

${ }^{3}$ Belman MJ, Gaesser GA. Ventilatory muscle training in the elderly. J Appl Physiol 1988; 64: 899-905

${ }^{4}$ Belman MJ, Shadmehr R. A target feedback device for ventilatory muscle training. J Clin Monit 1991; 7: 42-48

${ }^{5}$ Boussana A, Matecki S, Galy O, Hue O, Ramonatxo M, Le Gallais D. The effect of exercise modality on respiratory muscle performance in triathletes. Med Sci Sports Exerc 2001; 33: 2036-2043

${ }^{6}$ Boutellier U, Buchel R, Kundert A, Spengler C. The respiratory system as an exercise limiting factor in normal trained subjects. Eur J Appl Physiol 1992; 65: 347-353

7 Boutellier U, Piwko P. The respiratory system as an exercise limiting factor in normal sedentary subjects. Eur J Appl Physiol 1992; 64: $145-152$

${ }^{8}$ Caine MP, McConnell AK. Development and evaluation of a pressure threshold inspiratory muscle trainer for use in the context of sports performance. J Sports Engineering 2000; 3: 149-159

9 Chatham K, Baldwin J, Griffiths H, Summers L, Enright S. Inspiratory muscle training improves shuttle run performance in healthy subjects. Physiotherapy 1999; 85: 676-683

${ }^{10}$ Chatham K, Baldwin J, Oliver W, Summers L, Griffiths H. Fixed load incremental respiratory muscle training: A pilot study. Physiotherapy 1996; 82: 422-426

${ }^{11}$ Chen RC, Que CL, Yan S. Introduction to a new inspiratory threshold loading device. Eur Respir J 1998; 12: 208-211

${ }^{12}$ Cibella F, Cuttitta G, Romano S, Grassi B, Bonsignore G, Milic-Emili J. Respiratory energetics during exercise at high altitude. J Appl Physiol 1999; 86: $1785-1792$

${ }^{13}$ Clanton TL, Dixon G, Drake J, Gadek JE. Inspiratory muscle conditioning using a threshold loading device. Chest 1995; 87: 62 - 66

14 Dempsey JA. JB Wolffe memorial lecture. Is the lung built for exercise? Med Sci Sports Exerc 1986; 18: 143 - 155

${ }^{15}$ El-Manshawi A, Killian KJ, Summers E, Jones NL. Breathlessness during exercise with and without resistive loading. J Appl Physiol 1986; 61: $896-905$

${ }^{16}$ Fairbarn MS, Coutts KC, Pardy RL, McKenzie DC. Improved respiratory muscle endurance of highly trained cyclists and the effects on maximal exercise performance. Int I Sports Med 1991; 12: 66-70

${ }^{17}$ Gandevia SC, Killian KJ, Campbell EJ. The effect of respiratory muscle fatigue on respiratory sensations. Clin Sci (Colch) 1981; 60: 463-466

${ }^{18}$ Hanel B, Secher NH. Maximal oxygen uptake and work capacity after inspiratory muscle training: a controlled study. J Sports Sci 1991; 9: $43-52$

${ }^{19}$ Hanson P, Claremont A, Dempsey J, Reddan W. Determinants and consequences of ventilatory responses to competitive endurance running. J Appl Physiol 1982; 52: 615-623

${ }^{20}$ Hart N, Sylvester K, Ward S, Cramer D, Moxham J, Polkey MI. Evaluation of an inspiratory muscle trainer in healthy humans. Respir Med 2001; 95: 526-531

${ }^{21}$ Inbar O, Weiner P, Azgad Y, Rotstein A, Weinstein Y. Specific inspiratory muscle training in well-trained endurance athletes. Med Sci Sports Exerc 2000; 32: 1233 - 1237

22 Johnson BD, Babcock MA, Suman OE, Dempsey JA. Exercise-induced diaphragmatic fatigue in healthy humans. J Physiol (Lond) 1993; 460: $385-405$

${ }^{23}$ Johnson BD, Reddan WG, Seow KC, Dempsey JA. Mechanical constraints on exercise hyperpnea in a fit aging population. Am Rev Respir Dis 1991; 143: 968-977

${ }^{24}$ Keens TG, Krastins IR, Wannamaker EM, Levison H, Crozier DN, Bryan AC. Ventilatory muscle endurance training in normal subjects and patients with cystic fibrosis. Am Rev Respir Dis 1977; 116: 853-860 
25 Kohl J, Koller EA, Brandenberger M, Cardenas M, Boutellier U. Effect of exercise-induced hyperventilation on airway resistance and cycling endurance. Eur J Appl Physiol 1997; 75: 305-311

${ }^{26}$ Leith DE, Bradley M. Ventilatory muscle strength and endurance training. J Appl Physiol 1976; 41: $508-516$

27 Lenoir P, Jammes Y, Giry P, Rostain JC, Burnet H, Tomei C, Roussos C. Electromyographic study of respiratory muscles during human diving at 46 ATA. Undersea Biomed Res 1990; 17: 121 - 137

${ }^{28}$ Leveritt M, Abernethy PJ, Barry BK, Logan PA. Concurrent strength and endurance training. A review. Sports Med 1999; 28: $413-427$

${ }^{29}$ Markov G, Spengler CM, Knopfli-Lenzin C, Stuessi C, Boutellier U. Respiratory muscle training increases cycling endurance without affecting cardiovascular responses to exercise. Eur J Appl Physiol 2001; 85: $233-239$

${ }^{30}$ McClaran SR, Harms CA, Pegelow DF, Dempsey JA. Smaller lungs in women affect exercise hyperpnea. J Appl Physiol 1998; 84: 1872 1881

31 McConnell AK, Romer LM, Volianitis S, Donovan KJ. Re: Evaluation of an inspiratory muscle trainer in healthy humans (Respir Med 2001; 95: 526 - 531). Respir Med 2002; 96: 129-133

32 McMahon ME, Boutellier U, Smith RM, Spengler CM. Hyperpnea training attenuates peripheral chemosensitivity and improves cycling endurance. J Exp Biol 2002; 205: 3937 - 3943

33 Morgan DW, Kohrt WM, Bates BJ, Skinner JS. Effects of respiratory muscle endurance training on ventilatory and endurance performance of moderately trained cyclists. Int J Sports Med 1987; 8: 88 - 93

34 Powers SK, Coombes J, Demirel H. Exercise training-induced changes in respiratory muscles. Sports Med 1997; 24: 120-131

35 Ramirez-Sarmiento A, Orozco-Levi M, Guell R, Barreiro E, Hernandez N, Mota S, Sangenis M, Broquetas JM, Casan P, Gea J. Inspiratory muscle training in patients with chronic obstructive pulmonary disease: structural adaptation and physiologic outcomes. Am J Respir Crit Care Med 2002; 166: 1491 - 1497

${ }^{36}$ Redline S, Gottfried SB, Altose MD. Effects of changes in inspiratory muscle strength on the sensation of respiratory force. J Appl Physiol 1991; 70: $240-245$

37 Romer LM, Dempsey JA. The work of breathing during exercise: implications for performance. In: Aliverti A, Brusasco V, Macklem PT, Pedotti A (eds). Mechanics of Breathing: Pathophysiology, Diagnosis and Treatment. Milano, Italia: Springer-Verlag, 2002: $11-24$

\section{Zeitschrift IJSM sm109}

38 Romer LM, McConnell AK. Specificity and reversibility of inspiratory muscle training. Med Sci Sports Exerc 2003; 35: 237 - 244

39 Romer LM, McConnell AK, Jones DA. Effects of inspiratory muscle training upon recovery time during high intensity, repetitive sprint activity. Int J Sports Med 2002; 23: $353-360$

40 Romer LM, McConnell AK, Jones DA. Effects of inspiratory muscle training upon time trial performance in trained cyclists. J Sports Sci 2002; 20: $547-562$

41 Romer LM, McConnell AK, Jones DA. Inspiratory muscle fatigue in trained cyclists: effects of inspiratory muscle training. Med Sci Sports Exerc 2002; 34: 785 - 792

42 Smith K, Cook D, Guyatt GH, Madhavan J, Oxman AD. Respiratory muscle training in chronic airflow limitation: a meta-analysis. Am Rev Respir Dis 1992; 145: 533 - 539

43 Sonetti DA, Wetter TJ, Pegelow DF, Dempsey JA. Effects of respiratory muscle training versus placebo on endurance exercise performance. Respir Physiol 2001; 127: 185 - 199

44 Spengler CM, Roos M, Laube SM, Boutellier U. Decreased exercise blood lactate concentrations after respiratory endurance training in humans. Eur J Appl Physiol 1999; 79: 299-305

45 Stuessi C, Spengler CM, Knopfli-Lenzin C, Markov G, Boutellier U. Respiratory muscle endurance training in humans increases cycling endurance without affecting blood gas concentrations. Eur J Appl Physiol 2001; 84: $582-586$

46 Suzuki S, Sato M, Okubo T. Expiratory muscle training and sensation of respiratory effort during exercise in normal subjects. Thorax 1995; 50: $366-370$

47 Suzuki S, Yoshiike Y, Suzuki M, Akahori T, Hasegawa A, Okubo T. Inspiratory muscle training and respiratory sensation during treadmill exercise. Chest 1993; 104: 197-202

48 Volianitis S, McConnell AK, Koutedakis Y, McNaughton L, Backx K, Jones DA. Inspiratory muscle training improves rowing performance. Med Sci Sports Exerc 2001; 33: 803 -809

49 Williams JS, Wongsathikun J, Boon SM, Acevedo EO. Inspiratory muscle training fails to improve endurance capacity in athletes. Med Sci Sports Exerc 2002; 34: 1194-1198

50 Wilson RC, Jones PW. Influence of prior ventilatory experience on the estimation of breathlessness during exercise. Clin Sci (Colch) 1990; 78: $149-153$

51 Winkler G, Zifko U, Nader A, Frank W, Zwick H, Toifl K, Wanke T. Dosedependent effects of inspiratory muscle training in neuromuscular disorders. Muscle Nerve 2002; 23: 1257-1260

Author: There is no reference to Figure 1 in the text, please indicate where it should be placed. 